\title{
Caesium sputter ion source compatible with commercial SIMS instruments
}

\author{
S.F. Belykh ${ }^{\mathrm{a}, *}$, V.V. Palitsin ${ }^{\mathrm{a}}$, I.V. Veryovkin ${ }^{\mathrm{b}}$, A.P. Kovarsky ${ }^{\mathrm{c}}$, \\ R.J.H. Chang ${ }^{a}$, A. Adriaens ${ }^{d}$, M. Dowsett ${ }^{a}$, F. Adams ${ }^{\mathrm{e}}$ \\ ${ }^{\mathrm{a}}$ University of Warwick, Department of Physics, Warwick Coventry CV4 7AL, UK \\ ${ }^{\mathrm{b}}$ Argonne National Laboratory, Materials Science Division, Argonne, IL 60439, USA \\ ${ }^{\mathrm{c}}$ Ioffe Physical-Technical Institute, 194021 St. Petersburg, Russia \\ ${ }^{\mathrm{d}}$ Ghent University, Department of Analytical Chemistry, B-9000 Ghent, Belgium \\ ${ }^{\mathrm{e}}$ University of Antwerp, Department of Chemistry, 2610 Antwerp, Belgium \\ Received 12 September 2005; accepted 15 February 2006 \\ Available online 2 May 2006
}

\begin{abstract}
A simple design for a caesium sputter cluster ion source compatible with commercially available secondary ion mass spectrometers is reported. This source has been tested with the Cameca IMS $4 \mathrm{f}$ instrument using the cluster $\mathrm{Si}_{n}{ }^{-}$and $\mathrm{Cu}_{n}{ }^{-}$ions, and will shortly be retrofitted to the floating low energy ion gun (FLIG) of the type used on the Cameca 4500/4550 quadruple instruments. Our experiments with surface characterization and depth profiling conducted to date demonstrate improvements of analytical capabilities of the SIMS instrument due to the non-additive enhancement of secondary ion emission and shorter ion ranges of polyatomic projectiles compared to atomic ions with the same impact energy.
\end{abstract}

(C) 2006 Elsevier B.V. All rights reserved.

Keywords: Caesium sputter ion source; Non-additive sputtering; Atomic and cluster ion bombardment; Depth profiling; Floating low energy ion gun; Cluster-solid interaction

\section{Introduction}

One practical way to improve the analytical capabilities of existing SIMS instrumentation is to use cluster, rather than atomic, primary ions as projectiles. The bombardment of solids by small cluster ions with energies of a few $\mathrm{keV} /$ atom leads to a non-additive enhancement in sputtering [1] and in secondary ion yields [2,3], and a reduced penetration depth for probe atoms [4], which results in a reduction of ion beam-induced damage [5]. These fundamental features of "cluster-solid" interaction can significantly strengthen the SIMS analysis focused on the top-most surface atomic layers or/and on depth profiling because sputtering with cluster ions can substantially improve the elemental and molecular detection sensitivity, as well as the depth resolution.

An ion source which utilizes negative ions generated by bombarding a target with $\mathrm{keV} \mathrm{Cs}^{+}$ions is a suitable candidate to

\footnotetext{
* Corresponding author.

E-mail address: S.Belikh@warwick.ac.uk (S.F. Belykh).
}

be combined with commercially available SIMS instrumentation. The main advantage of such a source is a possibility of producing negative cluster ions from almost all elements. Negative cluster ions also have the useful property of minimizing charging effects when used for insulator analysis. Recently the first commercial ion source of the sputter-type (Peabody Scientific PSX-120) combined with the Cameca IMS $3 \mathrm{f}$ instrument has been used in SIMS analysis [4,6]. It was shown that the use of the $\mathrm{C}_{n}{ }^{-}$and $\mathrm{CsC}_{n}{ }^{-}$ions improves the capabilities of the SIMS instrument for a surface characterization and depth profiling. However, the authors of Refs. [4,6] have concluded that the source design has some disadvantages (such as the low efficiency of the ion source, the instability of the cluster ion beam, the contamination of the primary ion beam column by caesium) which limit the use of this design for the routine analysis.

In the present work, a simple design for a caesium sputter ion source free from above mentioned disadvantages and compatible with vacuum and ion-optical systems as well as with electronics of commercially available SIMS instruments is reported. This ion source has been tested with the Cameca IMS 
$4 \mathrm{f}$ instrument using cluster $\mathrm{Si}_{n}{ }^{-}$and $\mathrm{Cu}_{n}{ }^{-}$ions, and will shortly be retrofitted to the floating low energy ion gun (FLIG) of the type used on the Cameca 4500/4550 quadruple instruments. Results demonstrating how the use of cluster instead of atomic projectiles improves instrument capabilities in surface characterization and depth profiling are presented.

\section{Sputter ion source design combined with the Cameca IMS $4 f$ instrument}

A schematic drawing of the sputter ion source is shown in Fig. 1a. This device can be mounted on a Cameca IMS-4f instrument, without modification, in place of the duoplasmatron ion source. It consists of two vacuum housings: the source chamber and the adaptor for the connection of the source chamber with the SIMS instrument. The $\mathrm{Cs}^{+}$ion gun (1), the extraction electrode (2), and two heating filaments are mounted in the source chamber. The adaptor houses the shield electrode (3), the sputter target (4), and two electrodes of the immersion lens (5 and 6). The extraction electrode of the duoplasmatron source (7) was used as the third electrode of the immersion lens. The $\mathrm{Cs}^{+}$ion gun is an original $\mathrm{Cs}^{+}$ion source of the IMS-4f instrument where the ionizer has been modified to increase its efficiency. Sputter targets (4) were machined from $\mathrm{Si}$ and $\mathrm{Cu}$ in the shape of a $6 \mathrm{~mm}$ long rod with a circular ringed cone hole drilled through its center. Diameters of the entrance and exit holes of the sputter "cone" target were 5 and $1 \mathrm{~mm}$, respectively.

The operating principle of the ion source is as follows. Caesium chloride has been used as a source material for producing the $\mathrm{Cs}^{+}$ions. The filaments heat the container containing $\mathrm{CsCl}$ and the ionizer of the $\mathrm{Cs}^{+}$ion gun by the electron bombardment and the $\mathrm{CsCl}$ molecules evaporate and penetrate through the ionizer. Once these molecules contact the hot tungsten surface of the ionizer they dissociate, and surfaceionized $\mathrm{Cs}^{+}$ions are then accelerated to the target by a negative bias. The ionizer forms a slightly diverging and hollow $\mathrm{Cs}^{+}$ion beam with the current of $0.1-0.2 \mathrm{~mA}$ that sputters the "cone" target, but does not penetrate through its exit hole into the primary ion beam column. Negative ions, sputtered by the $\mathrm{Cs}^{+}$ projectiles, are extracted by the "push-pull" electric field [7], forming the cross-over behind the exit hole. The "push-pull" field is created into the "cone" space due to the superposition of electric fields applied between "shield electrode-target" and

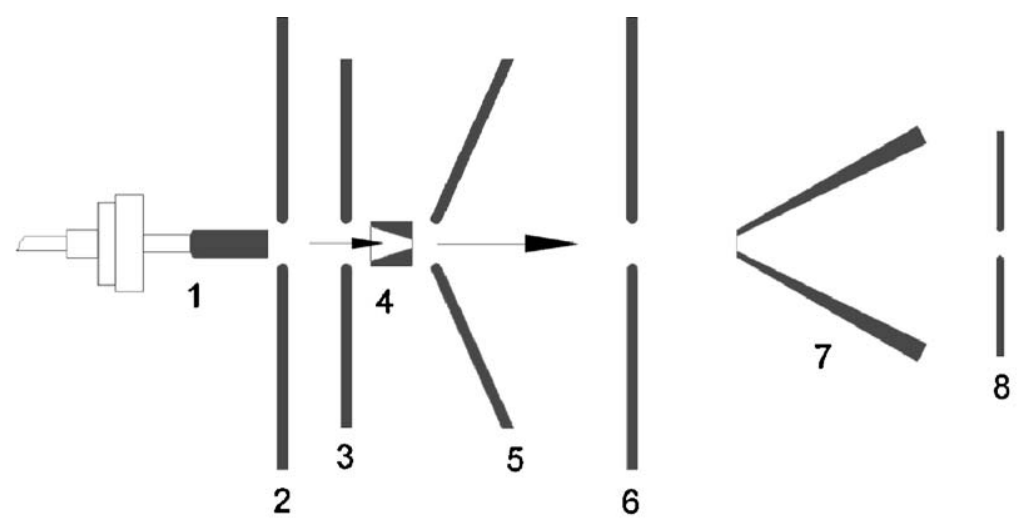

(a)

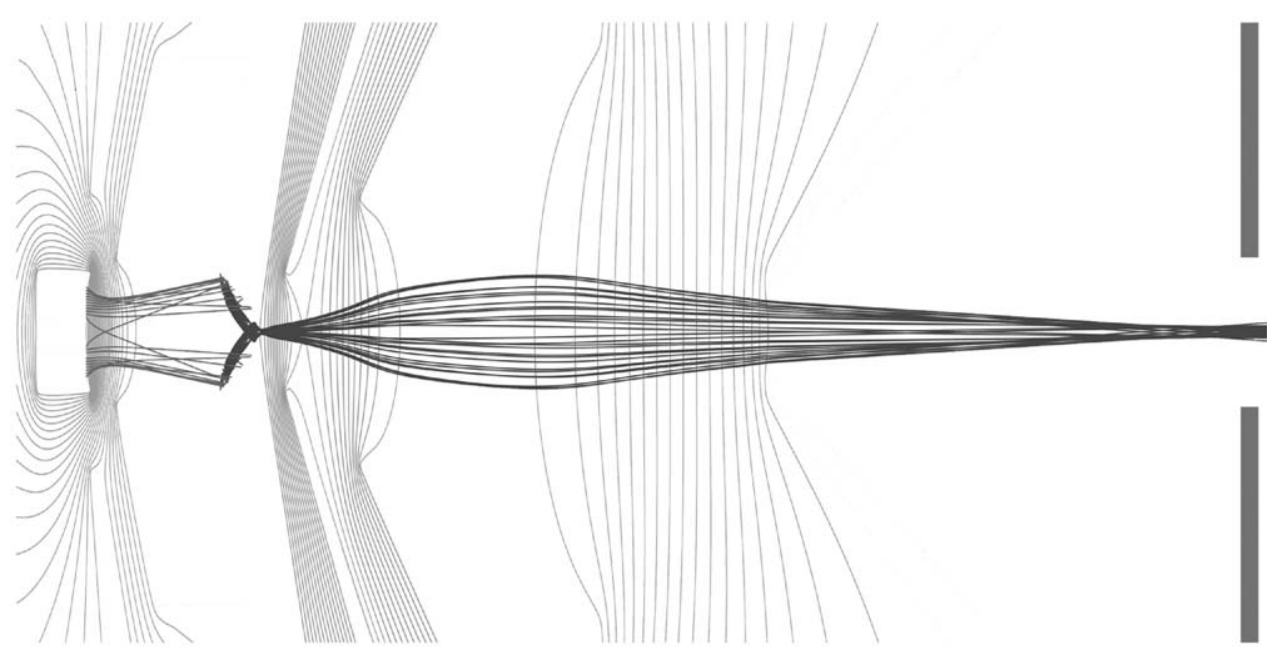

(b)

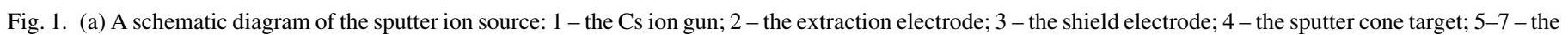

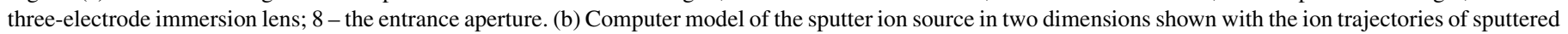
negative ions in the "sputter cone target-entrance aperture" space. 
"target-first electrode of the immersion lens". The negatively charged shield electrode (3) protects the ionizer from the damage due to the negative ion bombardment.

Optimal conditions for operating the Cameca IMS-4f instrument with the original duoplasmatron ion source occur when the image of the cross-over is formed near the entrance aperture (8) of the ion beam column. To operate the IMS-4f instrument with the sputter ion source, the immersion lens must form the image of the cross-over at the same position. However, positions and diameters of both the cross-over and its image depend on many ion-optical parameters (such as mutual arrangement, dimensions, shapes, and electrical potentials of the electrodes). Therefore, before the source design was implemented, it was simulated and improved using SIMION 3D ${ }^{\complement}$ computer code [8]. This permitted all-round examination of the design in order to optimize it for operation with the IMS4f instrument.

As an example, the simulated ion trajectories of negative cluster ions in the "sputter target-entrance aperture" space are shown in Fig. 1b. These results were used as a basis for the development of the sputter ion source design.

\section{Sputter ion source design combined with floating low energy ion gun (FLIG)}

The same ion source is currently being retrofitted to the floating low energy ion gun (FLIG). The FLIG holds the key to the delivery of high current density low energy ion beams at the sample [9], and such a device is essential for depth profiling with low energy, high erosion rate, and small area required in advanced SIMS applications. However, the final optical element in the FLIG column is a retarding immersion lens. Simple geometric optics shows that the magnification of the lens increases with the retardation (since the object space has a higher refractive index than the image space), which sets an ultimate limit on the column performance. The combination of a cluster source with the FLIG would allow for operation at lower retardation, with similar or lower impact energy per atom in the cluster, while at the same time accessing the potential for clusters to provide more efficient sputtering and secondary ion production.

Optimal conditions for operating the FLIG with its duoplasmatron ion source occur when the image of the cross-over is formed upstream of the variable aperture of the FLIG column. To operate the FLIG with the sputter ion source, both the adaptor and the immersion lens have been modified in order to form the image of the cross-over at the same position and the necessary optics were again designed using SIMION $3 \mathrm{D}^{\mathrm{C}}$. The ion trajectories corresponded to the situation when the sample is sputtered by the $1 \mathrm{keV} \mathrm{Cu}_{2}{ }^{-}$projectiles have been simulated. In this case, the cross-over diameter is of $0.2 \mathrm{~mm}$, the cross-over image is located near the variable aperture, and diameter of the ion beam spot on the sample is of $30 \mu \mathrm{m}$.

\section{Results}

The sputter ion source has been tested with the Cameca IMS 4f instrument using the cluster $\mathrm{Si}_{n}{ }^{-}$and $\mathrm{Cu}_{n}{ }^{-}$ions as projectiles. The following ion beam parameters were obtained.

Sputtering of the silicon "cone" target by the $7.5 \mathrm{keV} \mathrm{Cs}^{+}$ ions with a current of $60 \mu \mathrm{A}$ produces the $\mathrm{Si}_{m}{ }^{-}$ions $(m=1-6)$. After mass separating, the typical $\mathrm{Si}_{m}{ }^{-}$ion beam currents measured by the Faraday cup of the IMS $4 \mathrm{f}$ instrument were of $5.6 \mathrm{nA}, 2.8 \mathrm{nA}, 0.48 \mathrm{nA}$ and $85 \mathrm{pA}, 28 \mathrm{pA}, 6.3 \mathrm{pA}$, respectively, for $\mathrm{Si}^{-}, \mathrm{Si}_{2}{ }^{-}, \mathrm{Si}_{3}{ }^{-}$and $\mathrm{Si}_{4}{ }^{-}, \mathrm{Si}_{5}{ }^{-}, \mathrm{Si}_{6}{ }^{-}$ions.

In the same way, sputtering of the copper "cone" target by the $4.5 \mathrm{keV} \mathrm{Cs}^{+}$ions with a current of $100-150 \mu \mathrm{A}$ produces the $\mathrm{Cu}_{m}{ }^{-}$ions $(m=1-3)$. The $\mathrm{Cu}_{m}{ }^{-}$ion beam currents were of 2; 0.6; $0.9 \mathrm{nA}$, respectively, for $\mathrm{Cu}^{-}, \mathrm{Cu}_{2}$, and $\mathrm{Cu}_{3}{ }^{-}$ions.

The $\mathrm{Si}_{m}{ }^{-}$and $\mathrm{Cu}_{m}{ }^{-}$ion beams were focused on the sample surface to a spot size of $60 \mu \mathrm{m}$ providing current densities in the range of $0.04-0.08 \mathrm{~mA} / \mathrm{cm}^{2}$.

Table 1

The normalized yields $Y_{m}(m=1-3)$ of secondary ions sputtered from the InGaP sample by $12 \mathrm{keV} \mathrm{Si}_{m}{ }^{-}$projectiles $(m=1-3)$ and the ratios $Y_{2} / Y_{1}$ and $Y_{3} / Y_{1}$

\begin{tabular}{|c|c|c|c|c|c|}
\hline Species & $Y_{1}$ & $Y_{2}$ & $Y_{3}$ & $Y_{2} / Y_{1}$ & $Y_{3} / Y_{1}$ \\
\hline${ }^{31} \mathrm{P}^{+}$ & $1.3 \times 10^{-8}$ & $4.8 \times 10^{-8}$ & $8.3 \times 10^{-8}$ & 3.6 & 6.2 \\
\hline${ }^{31} \mathrm{P}_{2}^{+}$ & $2.4 \times 10^{-9}$ & $1.0 \times 10^{-8}$ & $3.9 \times 10^{-8}$ & 4.2 & 16.1 \\
\hline${ }^{69} \mathrm{Ga}^{+}$ & $2.1 \times 10^{-6}$ & $1.6 \times 10^{-5}$ & $8.1 \times 10^{-5}$ & 7.8 & 38.6 \\
\hline${ }^{69} \mathrm{Ga}_{2}^{+}$ & $1.7 \times 10^{-7}$ & $2.5 \times 10^{-7}$ & $5.3 \times 10^{-7}$ & 1.5 & 3.2 \\
\hline${ }^{115} \mathrm{In}^{+}$ & $1.7 \times 10^{-5}$ & $5.7 \times 10^{-5}$ & $3.1 \times 10^{-4}$ & 3.3 & 18.2 \\
\hline${ }^{12} \mathrm{C}^{+}$ & $2.0 \times 10^{-10}$ & $1.2 \times 10^{-9}$ & $1.4 \times 10^{-8}$ & 6.0 & 70.0 \\
\hline${ }^{23} \mathrm{Na}^{+}$ & $1.4 \times 10^{-8}$ & $4.0 \times 10^{-6}$ & $5.6 \times 10^{-6}$ & 285 & 400 \\
\hline${ }^{28} \mathrm{Si}^{+}$ & $2.6 \times 10^{-8}$ & $3.0 \times 10^{-7}$ & $2.5 \times 10^{-7}$ & 11.5 & 9.8 \\
\hline${ }^{28} \mathrm{Si}_{2}{ }^{+}$ & $1.6 \times 10^{-9}$ & $9.6 \times 10^{-9}$ & $2.8 \times 10^{-8}$ & 6.0 & 17.6 \\
\hline${ }^{28} \mathrm{Si}_{3}{ }^{+}$ & $4.8 \times 10^{-10}$ & $9.6 \times 10^{-10}$ & $3.4 \times 10^{-8}$ & 2.0 & 71.2 \\
\hline${ }^{39} \mathrm{~K}^{+}$ & $1.2 \times 10^{-8}$ & $7.7 \times 10^{-7}$ & $5.8 \times 10^{-6}$ & 66 & 497 \\
\hline${ }^{69} \mathrm{Ga}^{31} \mathrm{P}^{+}$ & $7.6 \times 10^{-9}$ & $3.6 \times 10^{-8}$ & $6.2 \times 10^{-8}$ & 4.7 & 8.1 \\
\hline${ }^{115} \mathrm{In}^{31} \mathrm{P}^{+}$ & $3.4 \times 10^{-8}$ & $1.1 \times 10^{-7}$ & $2.8 \times 10^{-7}$ & 3.3 & 8.3 \\
\hline${ }^{115} \mathrm{In}^{69} \mathrm{Ga}^{+}$ & $1.2 \times 10^{-6}$ & $1.9 \times 10^{-6}$ & $2.5 \times 10^{-6}$ & 1.6 & 2.0 \\
\hline${ }^{69} \mathrm{Ga}^{23} \mathrm{Na}^{+}$ & $8.8 \times 10^{-10}$ & $4.8 \times 10^{-9}$ & $5.1 \times 10^{-8}$ & 5.45 & 58.3 \\
\hline${ }^{28} \mathrm{Si}^{23} \mathrm{Na}^{+}$ & $4.8 \times 10^{-11}$ & $8.8 \times 10^{-10}$ & $4.6 \times 10^{-8}$ & 18.3 & 962 \\
\hline${ }^{69} \mathrm{Ga}^{1} \mathrm{H}^{+}$ & $4.8 \times 10^{-10}$ & $5.6 \times 10^{-9}$ & $1.8 \times 10^{-7}$ & 11.7 & 368 \\
\hline${ }^{31} \mathrm{P}^{16} \mathrm{O}^{+}$ & $6.4 \times 10^{-10}$ & $7.3 \times 10^{-8}$ & $4.0 \times 10^{-7}$ & 115 & 632 \\
\hline${ }^{69} \mathrm{Ga}^{16} \mathrm{O}^{+}$ & $8.4 \times 10^{-10}$ & $3.2 \times 10^{-9}$ & $3.4 \times 10^{-8}$ & 3.8 & 40.7 \\
\hline
\end{tabular}


The instability of these ion currents was less than $1-2 \%$. In such operating mode, the lifetime of the ion source typically exceeded $300-400 \mathrm{~h}$.

\subsection{Surface characterization of the InGaP sample}

The goal of the first test is the surface characterization of InGaP sample with $12 \mathrm{keV} \mathrm{Si}_{m}{ }^{-}$projectiles $(m=1-3)$. Mass spectra of sputtered positive ions consist of peaks corresponding to atomic and cluster ions of the target material, ions of elemental and molecular impurities, as well as heteronuclear ions. To compare the yields of the same ions produced by various projectiles, their peak intensities were normalized according to the ratio between the corresponding projectile ion currents. The normalized yields $Y_{m}(m=1-3)$ of secondary ions sputtered from the InGaP sample by $12 \mathrm{keV} \mathrm{Si}_{m}{ }^{-}$projectiles ( $m=1-3)$, as well as the ratios $Y_{2} / Y_{1}$ and $Y_{3} / Y_{1}$ are presented in Table 1. The comparison of these data shows clearly that, changing from the atomic $\mathrm{Si}^{-}$projectiles to the cluster $\mathrm{Si}_{2}{ }^{-}$and $\mathrm{Si}_{3}{ }^{-}$projectiles, results in a strong enhancement of the secondary ion yields.

\subsection{SIMS depth profiling}

SIMS depth profiling has evolved to encompass the use of low-energy ions $(<250 \mathrm{eV})$ to achieve the $\mathrm{nm}$-scale depth resolution as a result of a reduced ion range. However, the depth resolution cannot be improved further without significant loss of sensitivity due to the physical limits of sputtering by atomic ions (or light diatomic ones, such as $\mathrm{O}_{2}^{+}$). This contradiction might be avoided, using cluster ions of heavy and intermediate weight elements as projectiles. The advantages of such a method may be connected with various factors. (1) Enhanced depth resolution through a cluster energy partitioning. For a

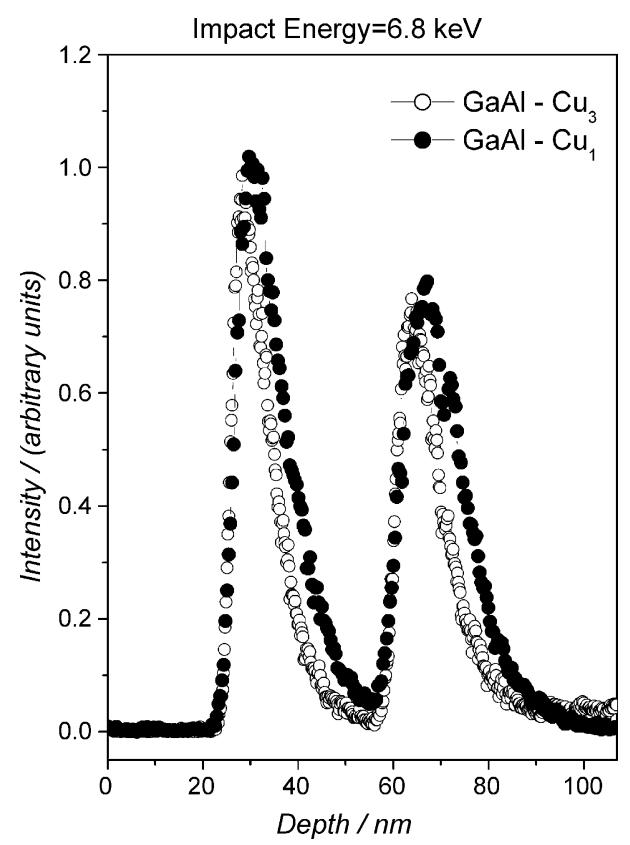

Fig. 2. Depth profiling of the GaAs sample with incorporated delta Al layers. given projectile energy, a heavier cluster constituent atom has a shorter ion range compared with an atomic ion. (2) Enhanced secondary ion yield through non-linear sputtering effects. (3) High erosion rates for low total cluster currents. (4) The small cluster energy spread. All these capabilities are exciting and have a potential to improve the depth resolution. However, the questions of how efficient cluster ions of heavy and intermediate weight elements can be in SIMS depth profiling still remain open.

The goal of the second test is to study depth profiling of the GaAs sample using the atomic $\mathrm{Cu}^{-}$and cluster $\mathrm{Cu}_{3}{ }^{-}$ projectiles with the same energy of $6.8 \mathrm{keV}$. The GaAs sample was grown by molecular beam epitaxy and contains two Al delta-layers $30 \mathrm{~nm}$ apart. The results of depth profiling, presented in Fig. 2, show that, compared with the $6.8 \mathrm{keV}$ $\mathrm{Cu}^{-}$projectiles, the use of the $2.26 \mathrm{keV} /$ atom $\mathrm{Cu}_{3}{ }^{-}$projectiles improves the depth resolution by a factor of 1.3 according to the FWHM definition.

\section{Concluding remarks}

The sputter ion source design was developed for commercially available SIMS instruments and has been tested with the Cameca IMS $4 \mathrm{f}$ instrument using the cluster $\mathrm{Si}_{n}{ }^{-}$and $\mathrm{Cu}_{n}{ }^{-}$ ions. So far, experiments with surface characterization and depth profiling conducted to date demonstrate the improvement of analytical capabilities of the SIMS instrument due to the nonadditive enhancement of secondary ion emission and shorter ion ranges of polyatomic projectiles compared to atomic ions with the same impact energy. One can anticipate that using much heavier cluster projectiles (such as $\mathrm{Au}_{m}{ }^{-}$cluster ions) with kinetic energies less than $1 \mathrm{keV}$ will permit the achievement of the nm-scale depth resolution without significant losses in sensitivity of the analysis. However, to realize this possibility, we will have not only to construct the necessary hardware for the cluster ion source in the FLIG configuration but also to conduct systematic studies of nonadditive sputtering of solids with the low energy heavy cluster projectiles. For now, this remains an open field awaiting nearfuture exploration.

\section{Acknowledgments}

The authors acknowledge support from EPSRC (Project GR/ S78919/01) and NATO (Project SfP 97.1929). IVV acknowledges support from the U.S. Department of Energy, BESMaterials Sciences, under Contract W-31-109-ENG-38. MGD acknowledges support from Cameca $\mathrm{GmbH}$.

\section{References}

[1] Y. Le Beyec, Int. J. Mass Spectrom. Ion Processes 174 (1998) 101.

[2] S.F. Belykh, B. Habets, U.Kh. Rasulev, A.V. Samartsev, L.V. Stroev, I.V. Veryovkin, Nucl. Instrum. Methods Phys. Res. B164-165 (2000) 809.

[3] S.F. Belykh, U.Kh. Rasulev, A.V. Samartsev, L.V. Stroev, A.V. Zinoviev, Vacuum 56 (2000) 257.

[4] G. Gillen, L. King, F. Chmara, J. Vac. Sci. Technol. A 17 (3) (1999) 845. 
[5] S.C.C. Wong, R. Hill, P. Blenkinsopp, N.P. Lockyer, J.C. Vickerman, Appl. Surf. Sci. 203-204 (2003) 219.

[6] G. Gillen, L. King, B. Freibaum, R. Lareau, J. Bennett, F. Chmara, J. Vac. Sci. Technol. A 19 (2001) 568.

[7] I.V. Veryovkin, W.F. Calaway, M.J. Pellin, Nucl. Instrum. Methods A 519 (2004) 353.
[8] D.A. S Dahl, SIMION 3D, Version 6, 43rd ASMS Conference on Mass Spectrometry and Allied Topics, Atlanta, GA, May 21-26, (1995), p. 717.

[9] M.G. Dowsett, N.S. Smith, R. Bridgeland, D. Richards, A.C. Lovejoy, P. Pedrick, in: A. Benninghoven (Ed.), Secondary Ion Mass Spectrometry SIMS X, Wiley and Sons Ltd., 1997, p. 367. 